\title{
Swinging jets
}

\author{
A. Bertsch $\odot,{ }^{1}$ A. Bongarzone $\odot,{ }^{2}$ E. Yim, ${ }^{2}$ P. Renaud $\odot,{ }^{1}$ and F. Gallaire ${ }^{2}$ \\ ${ }^{1}$ Microsystems Laboratory LMIS4, École Polytechnique Fédérale de Lausanne, Lausanne 1015, Switzerland \\ ${ }^{2}$ Laboratory of Fluid Mechanics and Instabilities, École Polytechnique Fédérale de Lausanne, \\ Lausanne 1015, Switzerland
}

(Received 31 July 2020; published 12 November 2020)

\begin{abstract}
This paper is associated with a video winner of a 2019 American Physical Society's Division of Fluid Dynamics (DFD) Gallery of Fluid Motion Award for work presented at the DFD Gallery of Fluid Motion. The original video is available online at the Gallery of Fluid Motion, https://doi.org/10.1103/APS.DFD.2019.GFM.V0036.
\end{abstract}

DOI: 10.1103/PhysRevFluids.5.110505

Liquid flows in microfluidic devices are generally laminar. As fluids are geometrically constrained in channels of submillimetric diameters, the Reynolds numbers associated with such liquid flows are generally low [1]. The most commonly described consequence is the difficulty of mixing fluids at such scales [2], as conditions where turbulence can develop are generally out of reach, leaving transport by molecular diffusion as the only available mechanism ensuring mixing. Despite these particularities in terms of fluid flows, a number of functions have been implemented in microfluidic devices and led to the development of laboratory-on-a-chip systems [3], which are seen as powerful tools for biology and healthcare applications [4,5].

Self-sustained pulsatile liquid flows are seldom observed at the microscale, and the scientific literature reports only a few examples of such oscillating microfluidic devices. Most of them are based on the Coandă effect and use either a feedback fluidic loop to generate pulsatile flows [6,7] or a jet impinging on a concave surface [8]. In the video presented here, pulsatile flows are generated at the microscale using a jet interaction scheme. The oscillations are self-sustained and result from the channel geometry only.

In the experiments presented in the video, a silicon chip containing two microchannels crossing at a right angle is used. Here the silicon microfluidic components were made using standard microfabrication techniques. The video shows devices with various geometries, but all of them have $100-\mu \mathrm{m}$-wide inlet channels and a height of $525 \mu \mathrm{m}$. Fluidic connections are made through a transparent polymeric cover slab, irreversibly bonded to the silicon. Dyed water enters the device by two inlets and is pushed into two long and narrow facing inlet channels, until it reaches a much larger transverse channel in which it creates two facing impinging jets with identical flow rates. Outlets are located at the ends of the large transverse channel, far from the intersection. Observations of the flow behavior are made using an inverted microscope and recorded with a high-speed camera. In parallel, direct numerical simulations were performed to solve the incompressible three-dimensional NavierStokes equations in the different oscillator geometries studied, using the open-source code NEK5000, which is based on a spectral element method [9]. For further details about the experimental setup and the numerical scheme see [10].

Published by the American Physical Society under the terms of the Creative Commons Attribution 4.0 International license. Further distribution of this work must maintain attribution to the author(s) and the published article's title, journal citation, and DOI. 

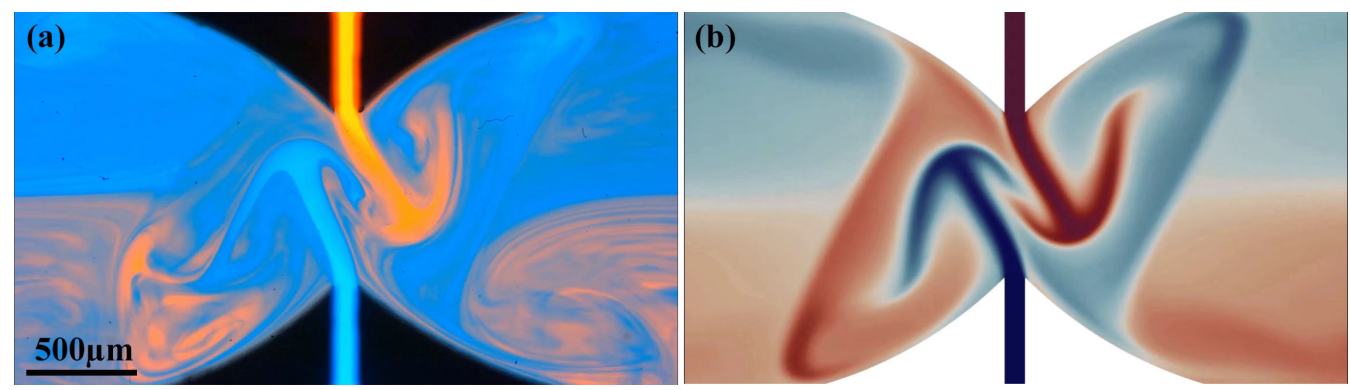

FIG. 1. Snapshot of the dye concentration fields in a micro-oscillator structure for $\mathrm{Re}=60$. The jets width is $100 \mu \mathrm{m}$, the distance between jets is $800 \mu \mathrm{m}$, the output channels width is $2000 \mu \mathrm{m}$, and the thickness of the device is $525 \mu \mathrm{m}$. (a) Experiment. The image results from the integration of the light crossing the full height of the structure. (b) Simulation of the dye concentration field in the plane of median height. The video associated with these images is available at https://doi.org/10.1103/APS.DFD.2019.GFM.V0036.

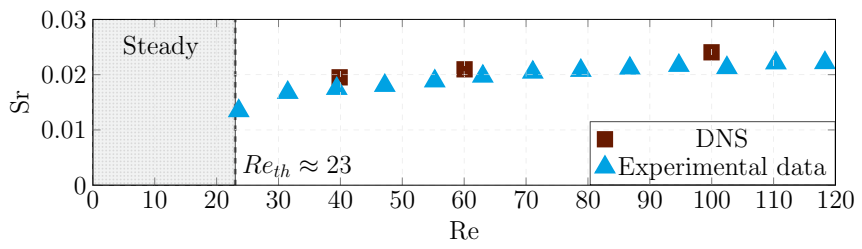

FIG. 2. Evolution of the nondimensional oscillation frequency or Strouhal number Sr with Reynolds number Re in the case of the geometric configuration described in Fig. 1, for which the instability threshold is approximatively $\mathrm{Re}_{t h} \approx 23$. For $\mathrm{Re}<\mathrm{Re}_{t h}$ the flow is steady.
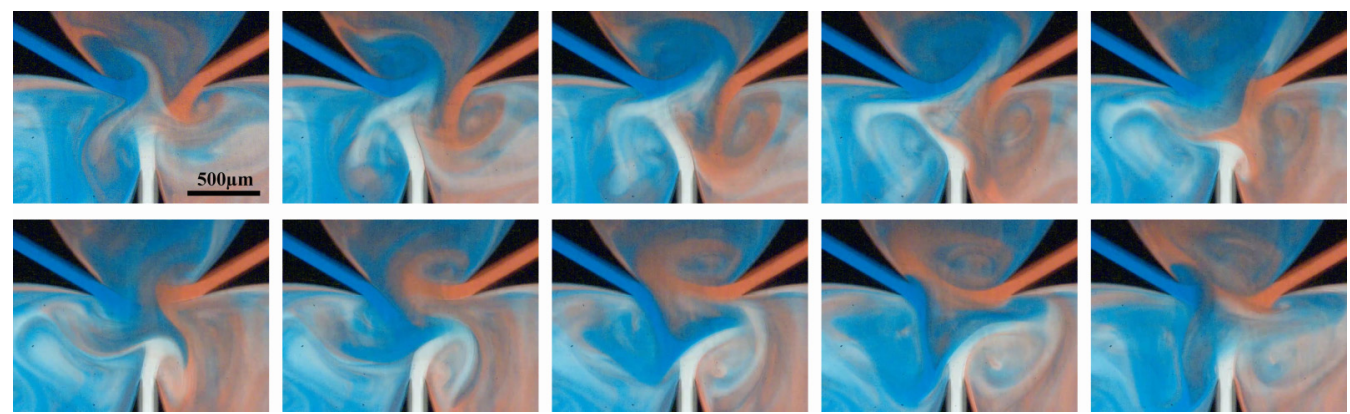

FIG. 3. Evolution of the dye concentration fields with time in a micro-oscillator structure with three inlet channels for $\operatorname{Re}=32$. The images are taken at regular time intervals during one oscillation (from left to right, top to bottom). The jets width is $100 \mu \mathrm{m}$, the three jets are placed at an angle of $120^{\circ}$ on a circle $800 \mu \mathrm{m}$ in diameter, the output channels width is $2000 \mu \mathrm{m}$, and the thickness of the device is $525 \mu \mathrm{m}$. The video associated with these images is available at https://doi.org/10.1103/APS.DFD.2019.GFM.V0036. 
The video shows that for appropriate channel geometries, when the flow rate of the facing jets increases, a threshold in Reynolds number $\mathrm{Re}_{t h}$, above which self-sustained oscillations of the jets do occur, is reached. The two flows oscillate in an antisymmetric way: They first bifurcate in opposite directions and later return towards one another until they collide, switch sides, and start a new oscillation. The frequency of the oscillations increases with the flow rate, and the range of stable oscillations depends on the shape of the output channel. A good match was obtained between the experimental observations and the results of the simulations as shown in Fig. 1 for the dye concentration field and in Fig. 2 in the case of the nondimensional oscillation frequency.

This self-oscillation phenomenon was also observed in other geometries involving more complex jet networks, including the one presented in Fig. 3, with three colliding jets. Similarly to the case with two inlets, the three jets oscillate and switch sides regularly. The physical mechanism at play in the self-sustained oscillations observed here is still unclear and an in-depth stability analysis needs to be conducted to elucidate it.

The authors gratefully acknowledge Lorenzo Siconolfi for his help with direct numerical simulations and Margaux Duchamp for cleanroom fabrication.

[1] D. Janasek, J. Franzke, and A. Manz, Scaling and the design of miniaturized chemical-analysis systems, Nature (London) 442, 374 (2006).

[2] A. D. Stroock, S. K. W. Dertinger, A. Ajdari, I. Mezić, H. A. Stone, and G. M. Whitesides, Chaotic mixer for microchannels, Science 295, 647 (2002).

[3] H. A. Stone, A. D. Stroock, and A. Ajdari, Engineering flows in small devices: Microfluidics toward a laboratory-on-a-chip, Annu. Rev. Fluid Mech. 36, 381 (2004).

[4] D. Figeys and D. Pinto, Lab-on-a-chip: A revolution in biological and medical sciences, Anal. Chem. 72, 330A (2000).

[5] P. Yager, T. Edwards, E. Fu, K. Helton, K. Nelson, M. R. Tam, and B. H. Weigl, Microfluidic diagnostic technologies for global public health, Nature (London) 442, 412 (2006).

[6] C. Xu, and Y. Chu, Experimental study on oscillating feedback micromixer for miscible liquids using the Coandă effect, AIChE J. 61, 1054 (2015).

[7] J. T. Yang, C. K. Chen, I. C. Hu, and P. C. Lyu, Design of a self-flapping microfluidic oscillator and diagnosis with fluorescence methods, J. Microelectromech. Syst. 16, 826 (2007).

[8] C. L. Sun and C. Y. Sun, Effective mixing in a microfluidic oscillator using an impinging jet on a concave surface, Microsyst. Technol. 17, 911 (2011).

[9] J. W. Lottes, P. F. Fischer, and S. G. Kerkemeier, NEK5000, available at http://nek5000.mcs.anl.gov (2008).

[10] A. Bertsch, A. Bongarzone, M. Duchamp, P. Renaud, and F. Gallaire, Feedback-free microfluidic oscillator with impinging jets, Phys. Rev. Fluids 5, 054202 (2020). 